\title{
A influência da equoterapia sobre o equilíbrio de crianças e adolescentes com transtorno do espectro autista: uma revisão integrativa da literatura
}

\author{
The influence of therapeutic riding on the balance of children and adolescents with autistic \\ spectrum disorder: an integrative literature review \\ La influencia de la conducción terapéutica en el equilibrio de niños y adolescentes con trastorno del \\ espectro autista: una revisión integrativa de la literatura
}

\section{Resumo}

O Transtorno do Espectro Autista (TEA) é definido como um distúrbio do neurodesenvolvimento, onde os primeiros sintomas surgem no início da infância. A fim de diminuir as complicações causadas pela doença, as crianças com TEA realizam sessões de fisioterapia e dentre os recursos utilizados, a Equoterapia vem se destacando como recurso complementar. O estudo foi realizado com objetivo de sistematizar as evidências cientificas acerca desse recurso, a fim de fornecer uma maior compreensão sobre a terapia, favorecendo subsídios para pesquisas futuras. Trata-se de uma revisão integrativa de literatura de caráter descritivo e com abordagem qualitativa, realizada através do levantamento bibliográficos nas bases de dados SciELO, PubMed e LILACS. Foram selecionados 5 artigos científicos, com base nos descritores "transtorno do espectro autista", "equoterapia assistida" e "equilíbrio postural". Todas as evidências apontaram conclusões consistentes sobre os benefícios da prática de equoterapia na saúde da criança e do adolescente com o TEA. Foi possível concluir que a prática regular da equoterapia é apropriada e indicada como plano terapêutico para crianças e adolescente com o TEA, porém, faz-se necessário mais estudos sobre a temática, visto que há uma escassez de referencial bibliográfico sobre benefícios da equoterapia no equilíbrio de pacientes infantojuvenis com TEA.

Palavras-chave: Transtorno do espectro autista; Equoterapia assistida; Equilíbrio postural; Pediatria. 


\begin{abstract}
Autism Spectrum Disorder (ASD) is defined as a neurodevelopmental disorder in which the first symptoms appear in early childhood. In order to reduce the complications caused by the disease, children with ASD undergo physiotherapy sessions, and among the resources used, horseback riding has stood out as a complementary resource. This study aimed to systematize the scientific evidence about this resource, in order to provide a better understanding of the therapy, favoring subsidies for future research. This is an integrative literature review with a descriptive and qualitative approach, carried out through bibliographic research in SciELO, PubMed and LILACS databases. 5 scientific articles were selected, based on the descriptors "autistic spectrum disorder", "assisted horseback riding" and "postural balance". All evidence pointed to consistent conclusions about the benefits of the practice of equine therapy on the health of children and adolescents with ASD. It was possible to conclude that the regular practice of equine therapy is appropriate and indicated as a therapeutic plan for children and adolescents with ASD; however, further studies on the theme are necessary, since there is a lack of bibliographic reference on the benefits of equine therapy on the balance of children and adolescents with ASD.
\end{abstract}

Keywords: Autism spectrum disorder; Equine assisted therapy; Postural balance; Pediatrics.

\title{
Resumen
}

El Trastorno del Espectro Autista (TEA) se define como un trastorno del neurodesarrollo, cuyos primeros síntomas aparecen en la primera infancia. Para reducir las complicaciones causadas por la enfermedad, los niños con TEA se someten a sesiones de fisioterapia y, entre los recursos utilizados, destaca la equitación como recurso complementario. El estudio se llevó a cabo con el fin de sistematizar la evidencia científica sobre este recurso, con el fin de proporcionar una mejor comprensión de la terapia, proporcionando subsidios para futuras investigaciones. Se trata de una revisión integradora de la literatura de carácter descriptivo con enfoque cualitativo, realizada mediante un relevamiento bibliográfico en las bases de datos SciELO, PubMed y LILACS. Se seleccionaron 5 artículos científicos, a partir de los descriptores "trastorno del espectro autista", "equinoterapia asistida" y "equilibrio postural". Todas las pruebas apuntaban a conclusiones consistentes sobre los beneficios de la práctica de la equinoterapia en la salud de los niños y adolescentes con TEA. Se pudo concluir que la práctica regular de la equinoterapia es adecuada e indicada como plan terapéutico para niños y adolescentes con TEA; sin embargo, son necesarios más estudios sobre el tema, ya que se carece de referencias bibliográficas sobre los beneficios de la equinoterapia en el equilibrio de los niños y adolescentes con TEA.

Palabras clave: Trastorno del espectro autista; Terapía asistida por caballos; Equilibrio postural; Pediatría.

\section{Introdução}

O Transtorno do Espectro Autista (TEA) é definido como um distúrbio do neurodesenvolvimento, onde os primeiros sintomas surgem no início da infância. Afeta a participação social e, consequentemente, o desenvolvimento psicomotor do indivíduo (Lee et al. 2015). Sabe-se que a presença dos comportamentos repetitivos, complexos e estereotipados são encontrados e considerados comuns no TEA, gerando prejuízos na qualidade de vida (Lázaro et al. 2016). Os movimentos podem envolver o corpo inteiro da criança ou adolescente, e se caracterizam por bruscas fugas para frente, balanceios e hiperextensão do pescoço (Lee et al. 2015; Lázaro et al. 2016).

A prevalência mundial da TEA é de 1 em cada 160 crianças, com sintomas iniciais que surgem na primeira infância e tendem a persistir na adolescência e na idade adulta (Campion et al. 2018; Cupertino et al. 2019). Enquanto alguns indivíduos com TEA podem viver de forma independente, outros tem deficiências severas e requerem cuidados e acompanhamento ao longo da vida adulta (Kalbassi et al. 2017; Campion et al. 2018; Pulikkan et al. 2018; Cupertino et al. 2019).

A falta de estabilidade postural se destaca nesse grupo, visto que a noção espaço-temporal e a sincronia muscular nos membros superiores e inferiores podem estar afetadas nesse grupo, influenciando no controle postural e no equilíbrio, causando consequentemente uma diminuição da realização das atividades funcionais (Tekola et al. 2020).

No intuito de trabalhar o controle postural e equilíbrio, sessões de fisioterapia são indicadas, e dentre os recursos utilizados, a Equoterapia vem se destacando como recurso complementar (Stins \& Emck, 2018; London et al. 2020; Baggio et al. 2021; Lima et al. 2021). Os estudos têm mostrado que a realização da Equoterapia pode trazer benefícios para a saúde do indivíduo, com efeito positivo na função muscular e sensitiva, gerando aumento reflexo vibratório tônico e, consequentemente causando um maior recrutamento de motoneurônio (Liporoni \& Oliveira, 2005). 
A Equoterapia pode ser descrita como um recurso terapêutico relacionado à reabilitação envolvendo animal: o cavalo, o que a diferencia do tratamento clínico convencional (Eckert, 2013; et al. 2021). Para os profissionais da saúde a Equoterapia seria um método complementar que utiliza o cavalo como facilitador e mediador dos processos terapêuticos e educacionais de reabilitação e socialização (Zamo \& Trentini, 2016).

Apesar das comprovações científicas sobre os efeitos da Equoterapia na saúde da criança e dos adolescentes, pouco se sabe sobre o efeito Equoterapia sobre equilíbrio de crianças e adolescentes com TEA. Dessa forma, o objetivo desse estudo é sistematizar as evidências científicas acerca desse recurso, a fim de fornecer uma maior compreensão sobre a terapia e favorecendo subsídios para pesquisas futuras.

\section{Metodologia}

Trata-se uma revisão integrativa da literatura de caráter descritivo e com abordagem qualitativa que visa possibilitar uma melhor compressão sobre os principais achados dos benefícios da Equoterapia sobre o equilíbrio de crianças e adolescentes com TEA. Sobre a metodologia de pesquisa referente ao estudo Mancini \& Sampaio (2006) diz:

Revisões da literatura são caracterizadas pela análise e pela síntese da informação disponibilizada por todos os estudos relevantes publicados sobre um determinado tema, de forma a resumir o corpo de conhecimento existente e levar a concluir sobre o assunto de interesse. Existem diversos tipos de estudos de revisão e cada um deles segue uma metodologia específica (Mancini \& Sampaio, 2006, p-1).

A revisão da literatura foi construída respeitando as seguintes etapas: 1) elaboração da pergunta norteadora; 2) busca na literatura; 3 ) coleta de dados; 4) análise crítica dos estudos incluídos; 5) discussão dos resultados; 6) apresentação da revisão. As perguntas que nortearam o presente estudo foram: "A Equoterapia pode melhorar o equilíbrio de crianças e adolescentes com TEA?", “Qual o impacto da Equoterapia na vida de criança e do adolescente com TEA?”.

Foi realizado levantamento bibliográfico nas bases de dados Scientific Electronic Library Online (SciELO), Public Medline (PubMed) e Literatura Latino-Americana e do Caribe em Ciências da Saúde (LILACS), sobre o assunto de interesse publicado no período de 2002 a 2020.

A seleção dos artigos foi realizada em abril e novembro de 2020, por todos os pesquisadores envolvidos de forma independentes utilizando os descritores nos idiomas inglês e português. Os critérios do sistema descritor utilizado nesta revisão foram realizados seguindo os Medical Subject Headings (MeSH) e Health Sciences Descriptors (DeCS) e o operador booleano, “AND”, foi utilizado. Os descritores selecionados para a busca dos artigos foram: Transtorno do Espectro Autista/Autism Spectrum Disorder AND Equoterapia Assistida/ EquineAssisted Therapy AND Equilíbrio postural/Postural Balance.

As evidências científicas foram selecionadas inicialmente pela leitura de título e resumo. Em sequência foi realizada a leitura de texto completo, para garantia ou não da adequação aos critérios de inclusão. Foi realizada uma síntese de cada estudo, trazendo suas principais informações sobre os mesmos.

Os critérios de inclusão dos artigos da presente revisão foram: publicações completas em periódicos nacionais e internacionais; revisão sistemática com ou sem meta-análise, estudo de caráter exploratório-descritivo; estudo de caráter exploratório e experimental e ensaios clínicos que contivessem informações sobre o papel da Equoterapia como recurso terapêutico em crianças e adolescentes com transtorno do espectro autista, bem como, as repercussões do equilíbrio.

Os critérios de não inclusão envolveram as publicações duplicadas, pesquisas que não apresentaram os desfechos de interesse e pesquisas que não utilizaram crianças e/ou adolescentes como amostra. A análise dos dados extraídos dos estudos foi realizada de forma descritiva, em duas etapas. Na primeira, foram identificadas informações quanto à localização do artigo, ano, metodologia e resultados principais. Na segunda etapa ocorreu a análise crítica dos artigos. 


\section{Resultados e Discussão}

Foram identificados 31 artigos nos bancos de dados da Scientific Electronic Library Online (SciELO), Public Medline (PubMed) e da Literatura Latino-Americana e do Caribe em Ciências da Saúde (LILACS). Após a aplicação dos critérios de inclusão, foram pré-selecionados 23 artigos para análise. 18 estudos foram excluídos por não apresentarem o desfecho de interesse, sobrando um total de 5 artigos para a realização do atual estudo. A Figura 1 mostra o processo de captação dos artigos.

Figura 1: Fluxograma da seleção dos estudos.

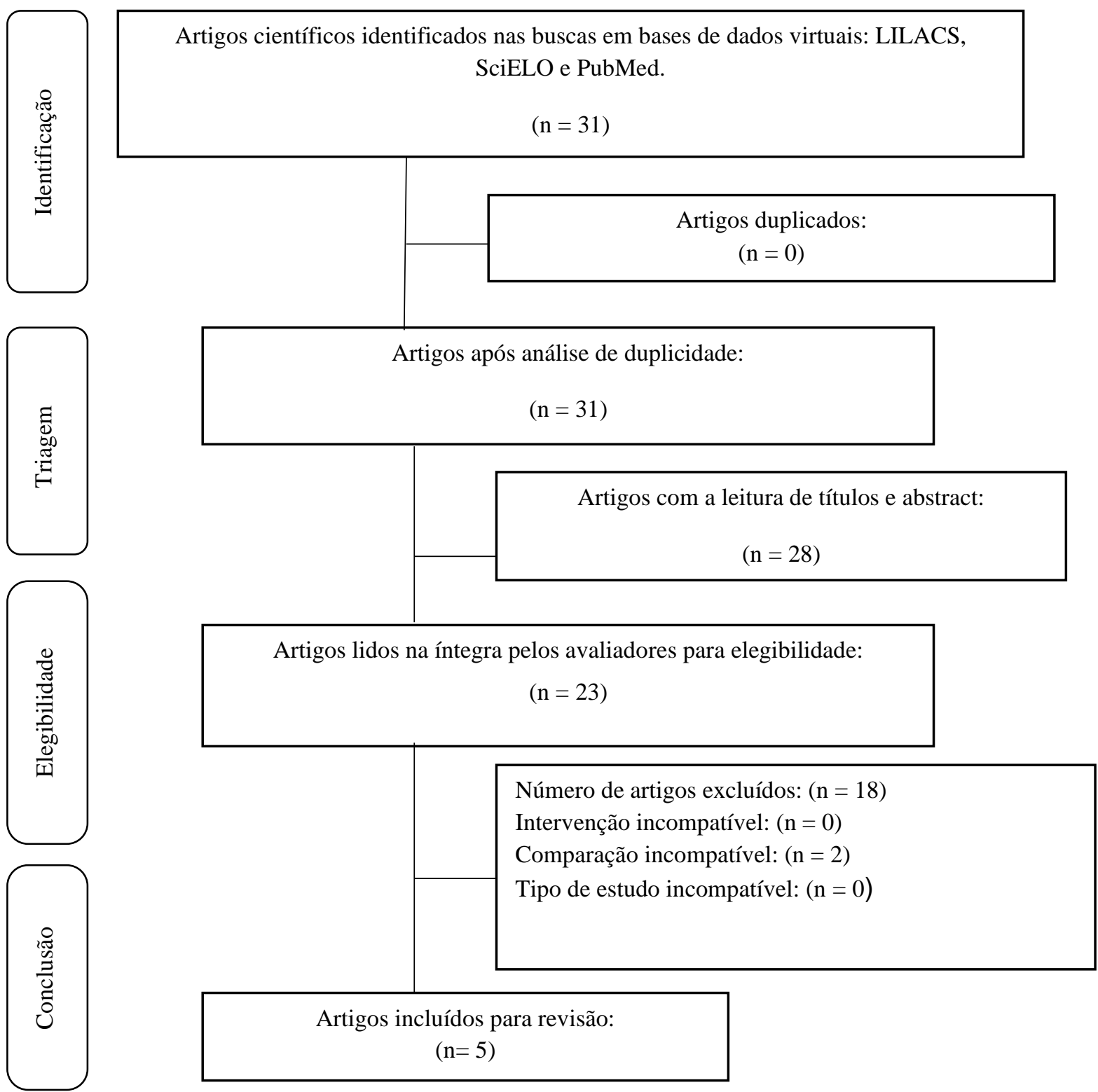

Fonte: Autores (2021).

Os artigos incluídos no estudo totalizaram uma amostra total de 428 participantes. Todos os estudos utilizaram a Equoterapia como recurso terapêutico e a idade dos voluntários variou de 2 a 16 anos. Ao analisar o período de publicação dos artigos, o maior número de artigos publicados foi em 2018 com três publicações, também foi encontrado uma publicação no ano de 2013 e 2017. Antes de participar da pesquisa, todos os voluntários apresentaram o diagnóstico de Transtorno do Espectro Autista. 
As sessões duraram de 38 a 67 minutos, onde o principal foco foi a estabilidade postural e o equilíbrio das crianças e dos adolescentes com o TEA. Dos 5 artigos, dois estudos foram revisões sistemáticas, um estudo foi de caráter exploratório com uma abordagem descritiva, um estudo de caráter experimental e um estudo de caso-controle. No Quadro 1 apresenta um breve resumo das principais informações dos estudos selecionados.

Quadro 1. Informações dos artigos selecionados.

\begin{tabular}{|c|c|c|c|}
\hline Autor/Ano & Tipo do estudo & Objetivo & Resultados \\
\hline $\begin{array}{l}\text { Srinivasan; Cavagnino \& } \\
\text { Baht, (2018) }\end{array}$ & Revisão sistemática & $\begin{array}{l}\text { Unir as evidências sobre o efeito } \\
\text { da equoterapia em crianças com } \\
\text { TEA. }\end{array}$ & $\begin{array}{l}\text { Os estudos mostraram um positivo na } \\
\text { função motora e cognitiva. }\end{array}$ \\
\hline $\begin{array}{l}\text { Sônego et } \\
\text { (2018) }\end{array}$ & $\begin{array}{l}\text { Um estudo de caráter } \\
\text { exploratório -descritivo. }\end{array}$ & $\begin{array}{l}\text { Avaliar o benefício que da } \\
\text { equoterapia proporciona para as } \\
\text { crianças de a } 2 \text { a } 11 \text { anos. }\end{array}$ & $\begin{array}{l}\text { Todos os voluntários apresentaram uma } \\
\text { melhora no equilíbrio. }\end{array}$ \\
\hline Ajzenman et al., (2013) & $\begin{array}{l}\text { Um estudo de caráter } \\
\text { experimental }\end{array}$ & $\begin{array}{l}\text { Verificar se a equoterapia pode } \\
\text { melhorar a participação das } \\
\text { crianças com TEA. }\end{array}$ & $\begin{array}{l}\text { Após a intervenção houve melhora no } \\
\text { balanço postural, equilíbrio e na } \\
\text { participação social. }\end{array}$ \\
\hline Harris \& Williams (2017) & $\begin{array}{l}\text { Um estudo de caso- } \\
\text { controle }\end{array}$ & $\begin{array}{l}\text { Relatar um estudo de caso- } \\
\text { controle de uma intervenção de } \\
\text { equitação para crianças com TEA }\end{array}$ & $\begin{array}{l}\text { Esses resultados indicam intervenção } \\
\text { melhora alguns aspectos do } \\
\text { funcionamento social e motor de crianças } \\
\text { e adolescente com TEA. }\end{array}$ \\
\hline Trzmiel et al., (2019) & $\begin{array}{l}\text { Uma } \\
\text { revisão sistemática com } \\
\text { meta-análise }\end{array}$ & $\begin{array}{l}\text { Avaliar a eficácia da equoterapia } \\
\text { em crianças e adolescentes com } \\
\text { TEA. }\end{array}$ & $\begin{array}{l}\text { Resultados encontraram um efeito positivo } \\
\text { para melhora do tônus muscular e no } \\
\text { equilíbrio dessa população. }\end{array}$ \\
\hline
\end{tabular}

Fonte: Autores (2022).

Os estudos tiveram um desenho metodológico adequado para o desenvolvimento da atual revisão. Todas as evidências apontaram conclusões consistentes sobre os benefícios da prática de equoterapia na saúde da criança e do adolescente com o TEA. Ao finalizar o seu estudo de caso, Harris \& Williams (2017), evidenciaram que essa população apresentou uma melhora no equilíbrio estático e dinâmico após o tratamento. A redução do estresse e a melhora do alinhamento corporal também foi observada nessa população.

Na revisão sistemática de Srinivasan el al. (2018), os artigos selecionados incluíram atividades como a hipoterapia e equitação terapêutica. Esses estudos utilizaram a manipulação intencional de movimento equino com base no raciocínio clínico do terapeuta, visto que o foco da hipoterapia é usar o cavalo como um recurso facilitador para promover os movimentos tridimensionais afim atingir os objetivos terapêuticos, tais como melhora do equilíbrio e habilidades de processamento sensorial.

Os autores dessa mesma pesquisa relatam que os pacientes om TEA podem apresentar alterações sensórios-motoras, causada pela dificuldade de modulação nas entradas sensoriais. Além da melhora da função muscular, o cavalo pode promover uma melhora da resposta sensitiva, favorecendo a transdução dos estímulos sensitivos (Srinivasan el al. 2018). 
Sônego et al (2018), afirma que os movimentos tridimensionais realizados pelos passos do cavalo levam às respostas motoras cruciais para o desenvolvimento de cada criança, tais como: regulação do tônus muscular, flexibilidade, equilíbrio e o aperfeiçoamento da coordenação motora, além de proporcionar estímulos proprioceptivos e vestibulares. O cavalo é o escolhido para esta prática por decorrência de ser o único animal capaz de produzir movimentos sequenciais que são os que mais se assemelham aos passos do ser humano.

A Equoterapia no tratamento de indivíduos com TEA está relacionada com atividades lúdico-esportivas e cinesioterapêuticas que possibilitam o desenvolvimento motor, cognição, aspectos emocionais e comportamentais. Essa prática permite que o indivíduo se sinta mais motivado no ensino e na aprendizagem, possibilitando maior disposição para o aprendizado, memorização e concentração, além dos aspectos motores, melhor noção espacial, equilíbrio, normalização do tônus muscular, aspectos sociais e maior valorização de si mesmo (Sônego et al. 2018).

Ajzenman et al. (2013), realizaram um programa de hipoterapia em crianças portadoras de TEA com idade variando de 5-12 anos. Através da hipoterapia, foi realizada a estimulação das atividades básicas e atividades mais avançadas para desafiar o equilíbrio postural, a comunicação, habilidades sociais e toque. A fim de proporcionar mudanças no posicionamento e na funcionalidade dos membros superiores. Os resultados demonstraram aumento da estabilidade postural, interação social, participação das atividades funcionais, além do progresso das habilidades motoras.

Os artigos selecionados para a revisão Trzmiel et al. (2019), totalizaram uma amostra de 380 participantes. A idade dos participantes dos estudos variou de 3 a 16 anos, esses participantes eram de ambos os sexos, porém com maior predominância o sexo masculino, correspondendo a $79 \%$ e o feminino $21 \%$. Todos os voluntários tinham o diagnóstico confirmado de TEA. Os estudos dessa revisão sistemática demostraram eficácia da equoterapia, no que diz respeito ao movimento rítmico, funções físicas do corpo, função muscular e, consequentemente melhorando do equilíbrio estático e dinâmico (Trzmiel et al. 2019). A melhora da função muscular também foi encontrada no estudo de Harris e Willians (2017), em seu estudo de caso controle.

Devido à grande quantidade de estímulos que é proporcionado ao praticante, através do uso do cavalo como um instrumento cinesioterapêutico. Pode-se perceber que a equoterapia ainda é um método novo em nosso meio, contudo, podemos colher muitos benefícios com essa prática (Harris \& Willians, 2017).

Nos estudos analisados, os pais dos voluntários relataram um efeito positivo na qualidade de vida dos mesmos. Diante disso, torna-se evidente destacar os resultados positivos que os praticantes com TEA estão obtendo com a equoterapia, principalmente, devido à grande quantidade de estímulos que favorece a neuroplasticidade cerebral. Com base nos estudos revisados é possível confirmar que a equoterapia é uma terapia eficaz para melhorar o equilíbrio dessa população, porém faz-se necessário novos ensaios clínicos para mostrar os resultados mais fidedignos.

\section{Considerações Finais}

O presente estudo constatou o papel da equoterapia no tratamento de crianças com TEA, elencando que as atividades assistidas por cavalos geram efeitos positivos e significativos no desenvolvimento das crianças e do adolescente com TEA, principalmente no que se refere à equilíbrio e motricidade. Foi possível concluir que a prática regular da equoterapia é apropriada e indicada como plano terapêutico para crianças e adolescente com o TEA. Fazendo-se necessário mais estudos sobre a temática, visto que poucos estudos mostraram os benefícios da equoterapia sobre o equilíbrio de pacientes infantojuvenis com TEA.

\section{Referências}

Ajzenman, H. F., Standeven, J. W., \& Shurtleff, T. L. (2013). Effect of hippotherapy on motor control, adaptive behaviors, and participation in children with autism spectrum disorder: a pilot study. The American journal of occupational therapy: official publication of the American Occupational Therapy Association, 67(6), 653-663. https://doi.org/10.5014/ajot.2013.008383. 
Baggio, G., Bolfe, K. D., Werkhausen, N.., \& Mello, P. C. (2021). Hippotherapy: therapeutic and educational interventions with people with disabilities and with Autistic Spectrum Disorder. Research, Society and Development, 10(13), e438101321353. https://doi.org/10.33448/rsd-v10i13.21353.

Campion, D., Ponzo, P., Alessandria, C., Saracco, G. M., \& Balzola, F. (2018). The role of microbiota in autism spectrum disorders. Minerva gastroenterologica e dietologica, 64(4), 333-350. https://doi.org/10.23736/S1121-421X.18.02493-5

Cupertino, M. do C., Resende, M. B., Veloso, I. de F., Carvalho, C. A. de, Duarte, V. F., \& Ramos, G. A. (2019). Autism spectrum disorder: a systematic review about nutritional aspects and gut-brain axis. ABCS Health Sciences, 44(2). https://doi.org/10.7322/abcshs.v44i2.1167

Eckert D. (2013) Equoterapia como recurso terapêutico: análise eletromiográfica dos músculos reto do abdômen e paravertebral durante a montaria. 57f. [Dissertação]. Centro Universitário Univates, Lajeado.

Harris, A., \& Williams, J. M. (2017). The Impact of a Horse Riding Intervention on the Social Functioning of Children with Autism Spectrum Disorder. International journal of environmental research and public health, 14(7), 776. https://doi.org/10.3390/ijerph14070776

Kalbassi, S., Bachmann, S. O., Cross, E., Roberton, V. H., \& Baudouin, S. J. (2017). Male and Female Mice Lacking Neuroligin-3 Modify the Behavior of Their Wild-Type Littermates. eNeuro, 4(4), ENEURO.0145-17.2017. https://doi.org/10.1523/ENEURO.0145-17.2017

Lázaro, C. P., Pondé, M. P., Rodrigues L. E. (2016) Opioid peptides and gastrointestinal symptoms in autism spectrum disorders. Braz J Psychiatry. 2016 JulSep;38(3):243-6.

Lee, P. F., Thomas, R. E., \& Lee, P. A. (2015). Approach to autism spectrum disorder: Using the new DSM-V diagnostic criteria and the CanMEDS-FM framework. Canadian family physician Medecin de famille canadien, 61(5), 421-424.

Lima, M. B. de, Algeri, S.., Silva, P. A. da, Silva, M. P. da, Oliveira, N. B. C. de., \& Viana, A. C. W. (2021). Horse therapy benefits in children with cerebral palsy: integrative review. Research, Society and Development, 10(2), e29810212506. https://doi.org/10.33448/rsd-v10i2.12506.

Liporoni, G. L., Oliveira, A. P. R. (2005). Equoterapia como tratamento alternativo para pacientes com seqüelas neurológicas. Revista Científica da Universidade de Franca. Jan; 5(1): 1-6.

London, M. D., Mackenzie, L., Lovarini, M., Dickson, C., \& Alvarez-Campos, A. (2020). Animal Assisted Therapy for Children and Adolescents with Autism Spectrum Disorder: Parent perspectives. Journal of autism and developmental disorders, 50(12), 4492-4503. https://doi.org/10.1007/s10803-020-04512-5.

Mancini, M. C., Sampaio, S. R. (2006) Quando o objeto de estudo é a literatura: estudos de revisão. Rev. bras. fisioter. 10(4); 01.

Mello, P. C., Scortegagna , H. de M., Fioreze, C., Palmeiras, G. de B., \& Bona, C. C. (2021). Riding therapy on the perception of parents/mothers of autistic. Research, Society and Development, 10(11), e145101119495. https://doi.org/10.33448/rsd-v10i11.19495

Pulikkan, J., Maji, A., Dhakan, D. B., Saxena, R., Mohan, B., Anto, M. M., Agarwal, N., Grace, T., \& Sharma, V. K. (2018). Gut Microbial Dysbiosis in Indian Children with Autism Spectrum Disorders. Microbial ecology, 76(4), 1102-1114. https://doi.org/10.1007/s00248-018-1176-2

Sônego, G. L., Cavalante, J. V. M., Sousa, L. C., Quaggio, C., M. (2018) Contributions of equotherapy to the development of disabled children: an interdisciplinary approach. Rev. Salusvita, 37(3): 653-670.

Srinivasan, S. M., Cavagnino, D. T., \& Bhat, A. N. (2018). Effects of Equine Therapy on Individuals with Autism Spectrum Disorder: A Systematic Review. Review journal of autism and developmental disorders, 5(2), 156-175. https://doi.org/10.1007/s40489-018-0130-z.

Stins, J. F., \& Emck, C. (2018). Balance Performance in Autism: A Brief Overview. Frontiers in psychology, 9, 901. https://doi.org/10.3389/fpsyg.2018.00901

Tekola, B., Girma, F., Kinfe, M., Abdurahman, R., Tesfaye, M., Yenus, Z., WHO CST Team, Salomone, E., Pacione, L., Fekadu, A., Servili, C., Hanlon, C., \& Hoekstra, R. A. (2020). Adapting and pre-testing the World Health Organization's Caregiver Skills Training programme for autism and other developmental disorders in a very low-resource setting: Findings from Ethiopia. Autism: the international journal of research and practice,24(1), 51-63. https://doi.org/10.1177/1362361319848532

Trzmiel, T., Purandare, B., Michalak, M., Zasadzka, E., \& Pawlaczyk, M. (2019). Equine assisted activities and therapies in children with autism spectrum disorder: A systematic review and a meta-analysis. Complementary therapies in medicine, 42, 104-113. https://doi.org/10.1016/j.ctim.2018.11.004.

Zamo, R. S., \& Trentini C., R. (2016). Psychological assessment and therapeutic riding: a systematic review. Psicol. teor. prat. 2016, 18(3):81-97. 